\title{
Towards Extending Sensor Node Lifetime with Printed Supercapacitors
}

\author{
Andrey Somov ${ }^{1}$, Christine C. Ho $^{2}$, Roberto Passerone ${ }^{3}$, \\ James W. Evans ${ }^{4}$, and Paul K. Wright ${ }^{4,5}$ \\ ${ }^{1}$ CREATE-NET, Trento, Italy \\ andrey. somovecreate-net.org \\ ${ }^{2}$ Imprint Energy, Berkeley, CA, USA \\ chi@imprintenergy. com \\ ${ }^{3}$ University of Trento, Trento, Italy \\ roberto.passerone@unitn. it \\ ${ }^{4}$ University of California, Berkeley, CA, USA \\ evans@berkeley.edu, pwright@bmi.berkeley.edu \\ ${ }^{5}$ Center for Information Technology Research in the Interest of Society, Berkeley, CA, USA
}

\begin{abstract}
The realization of completely autonomous wireless sensor networks (WSN) has been hindered with difficulties in implementing a truly "perpetual" power supply. Typically, the generic power supply for a sensor node is a battery, which is limited in cycle life. Current research in energy scavenging technology, along with the use of innovative energy storage devices such as supercapacitors, has demonstrated the improvement of sensor node lifetime characteristics. In this paper, we present the experimental results on a novel electrochemical supercapacitor (with improved electrical characteristics) manufactured using a "direct write" deposition tool. This technology allows one to print the supercapacitors with capacitances above $40 \mathrm{mF} / \mathrm{cm}^{2}$ directly on board of a sensor node covering any unoccupied surface area. Experiments on the printed storage chrarging with (AC) and (DC) based ambient energy via a specially developed generic energy scavenging module (ESM), and 24-hours deployment with a typical sensor node have showed promise towards extending sensor node lifetime.
\end{abstract}

Keywords: wireless sensor network, energy scavenging, direct write, electrochemical capacitor, power supply and management.

\section{Introduction}

A wireless sensor network (WSN) consists of a number of wireless nodes able to interact with the environment and among themselves inside a network. If the network is deployed in a difficult-to-access, area it is a non trivial task to change the batteries which are the typical power supply for the sensor node, or to replenish another power supply source, reducing the lifetime of the network. Energy scavenging technology resolves partially the problem of sensor node lifetime [3], [21]. However, the type of 
ambient source availability, e.g. (AC) or (DC) based, quite often constrains the WSN deployment in various environments. Moreover, the sensor node has to contain a sufficiently large energy buffer for the scavenged energy.

In this paper we aim to improve the lifetime of the sensor node using printed supercapacitors as an energy buffer for the scavenged energy. For the experimentation purpose we implement an Energy Scavenging Module (ESM) able to accept both AC (such as vibrations), and DC (such as solar panels) environmental energy sources. The scavenged energy is stored in two energy buffers with complementary characteristics. The primary buffer consists of a supercapacitor, which has more limited storage capacity, but does not suffer as dramatically from degradation due to high rate discharging; the secondary buffer is a traditional rechargeable lithium-ion battery, with a large capacity but limited cycle life. The novel aspect of the design is the use of ink printing fabrication technology that allows the supercapacitor to be deposited in the empty spaces of the node board, using a "direct write" printing technique. This solution allows the node to have a compact layout and increases the on-board energy storage capacity. This paper focuses on the experimentation with printed supercapacitors, so we will not be concerned with the design of the harvesters that are covered elsewhere in the literature [21].

After the background information in electrochemical materials deposition in Section 2, we discuss lifetime problem in WSN (in particular the energy properties of WSN) and present the design goals (in terms of prolonged long-term operation) for our experimental system in Section 3. Our experimental system implementation is described in Section 4. The experimental results are demonstrated in Section 5. The review of the recent development of wireless sensor node platforms utilizing energy scavenging technology with a single or dual energy buffer is presented in Section 6. We conclude by describing the obtained results and future work.

\section{Background: Method of Electrochemical Materials Deposition}

In this section we describe a printing method for fabricating electrochemical capacitors directly onto a substrate.

Recent research improvements in carbon electrode and electrolyte material have resulted in a growing interest in carbon electrochemical capacitors as suitable devices for both solitary and load leveling energy buffer configurations [11]. Electrochemical capacitors store energy via electrostatic arrangements at the interface between carbon electrode particles and electrolyte ions. The combination of high surface area carbon electrode materials along with electrolytes stable under large electrochemical potentials results in substantial energy storage capabilities within a small device volume [12].

The utilization of organic solvents [13], and more recently novel ionic liquids [14] as supercapacitor electrolytes has enabled greater electrochemical stability over larger potential ranges than aqueous electrolytes, which are only stable up to $\Delta 1 \mathrm{~V}$. As a result, energy density, being a function of the square of the potential difference 
achievable in a capacitor, increases significantly. Ionic liquids especially are interesting because they effectively remain liquid at room temperature with negligible vapor pressure, and therefore are not volatile [15]. Besides being safe and requiring less hermetic packaging, these novel liquids provide an interesting opportunity when incorporated with polymer gels to form solid-like films that maintain liquid-like properties. We have developed a gel electrolyte with high mechanical strength and good ionic conductivity by incorporating 1-butyl-3-methylimidazolium tetrafluoroborate $\left(\mathrm{BMIM}^{+} \mathrm{BF}_{4}^{-}\right)$ionic liquid with polyvinlydene fluoride (PVDF) polymer [16]. Using this gel electrolyte, solid-state electrochemical capacitors can be deposited utilizing direct write solutions processing.

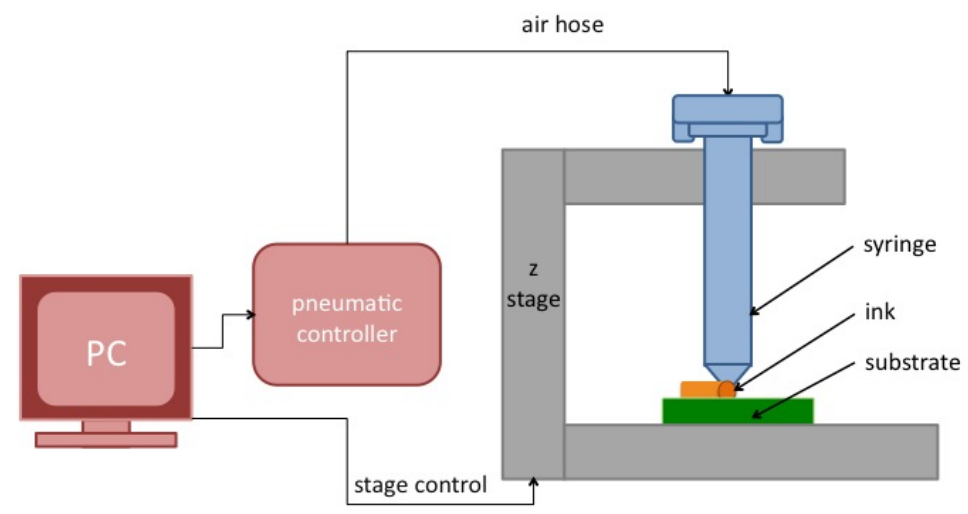

Fig. 1. Schematic of direct write, pneumatic dispenser printer

Direct write solutions processes are typically simple methods for depositing materials additively onto a substrate in ambient environments and in room temperature conditions. They provide a suitable alternative to standard thin film microfabrication techniques for devices that benefit from thick films or non-planar geometries such as electrochemical capacitors. We have developed a pneumatic dispenser printing tool which extrudes slurries and solutions onto a substrate mounted on a 3-axis stage with 5 micron accuracy (Fig. 1). Because the deposition of solutions is gentle, films of different compositions can be deposited in a subsequent manner, and devices can be built. Several direct write processes have been used to deposit similar structures [17], [18], but our printer provides the added benefits of reducing energy inputs and waste generated, as well as being able to deposit material around existing components, effectively utilizing any open space on a substrate. Earlier results have shown this method to be both cost effective and scalable for the mass production [blind review].

\section{$3 \quad$ Lifetime Problem}

In this section we present the relevant energy properties of sensor networks that influenced their lifetime and discuss the design goals for our experimental system. 


\subsection{Energy Properties of WSN}

First of all, sensor nodes are energy-constrained devices, so that energy aspects are one of the primary concerns of WSN developers. Second, sensor nodes typically perform some periodic tasks, e.g., sensing and communication, defined by user. The nodes which operate at a low duty cycle of activation can significantly increase their lifetime. In our prototype this is an important factor, since the current hardware limitations do not allow the printed supercapacitors to be used in communication mode, because of constraints on the available current, but only during low energy sensing and sleep modes.

Third, the nodes which adopt both primary (supercapacitor based) and secondary (battery based) energy buffers are recommended to use the primary one first, due to its higher leakage current and increased number of charge-discharge cycles.

Finally, the nodes can be deployed in difficult-to-access areas which constraints the access to the WSN and, therefore, the battery replacement becomes complicated. The energy scavenging technology can be applied for improved long-term operation of the sensor nodes.

\subsection{Design Goals}

Our main design goal is to provide a sensor node with a 'perpetual' power supply. To achieve this main objective we have defined the following subgoals for our experimental system:

- take what is available: the ESM should support constant ambient energy harvesting, i.e. during the daytime the module can harvest solar radiation (DC mode) since it provides the highest energy density [21] whereas during night it can switch, for example, to vibrations' collection (AC mode);

- power management: energy is wasted any time voltage needs to be converted. In an ideal case, energy from a buffer would be applied directly to the electronic device. In reality, however, additional circuits are required to manage this conversion to meet voltage, noise and other characteristics of the particular device. These circuits are power consumers as well as the electronic device. Apart from the 'management' activities, the goal of the power management circuits is to be as energy neural as possible;

- smart choice of system power supply. The ESM has three available power supplies - a harvesting component, the li-ion battery, and printed supercapacitors. This goal consists in choosing the best power supply at any time based on the node lifetime requirements and the characteristics of each power supply and available ambient energy. The main power supply in the system should be a harvesting component. We assume that the ambient energy source is essentially inexhaustible (for instance, a solar panel has a typical lifecycle from 5 to 7 years). While the ambient source is available the module supplies the sensor node with ambient energy. As soon as the ambient source becomes unavailable, the system requests energy from the printed supercaps ( $>120,000$ charge-discharge cycles). If the supercaps are sufficiently charged to supply the sensor node, and if it, in turn, is able to operate in a 
sleep or low-energy mode (due to the maximum discharge current of $\sim 2 \mathrm{~mA}$ supported by the supercapacitors), the supercaps become the main power supply for the node. When two previous sources are unavailable, the Li-ion battery $(\sim 300-500$ charge discharge cycles) starts to supply the sensor node. At the same time the system requests a harvesting component as soon as it becomes available. This algorithm enables to keep the long-term operation of the energy buffers and increase the entire system lifetime.

- trade-off between improved energy buffer and the size of ESM: since the capacity of printed supercaps depends on the surface of ESM available for printing a user should follow this trade-off. In this paper, however, we have designed a prototype of ESM and, therefore, mainly focused on 'proof of concepts' rather then capacity/size aspects.

\section{Experimental System}

This section presents the experimental system which is used in this work for the experimentation. The system consists of the printed supercapacitors, ESM, and the sensor node. Since we use off-the-shelf node we cover only the implementation of the printed supercapacitors and ESM.

\subsection{System Architecture}

The ESM requirements are the following: the module is to harvest the ambient (AC or DC based) energy, store it in two energy buffers, or supply a sensor node directly from a harvesting component or by energy stored in the energy buffers. A functional block diagram of the ESM is depicted in Figure 2. The parts inside the shaded area are referred to the ESM except for the printed supecapacitors.

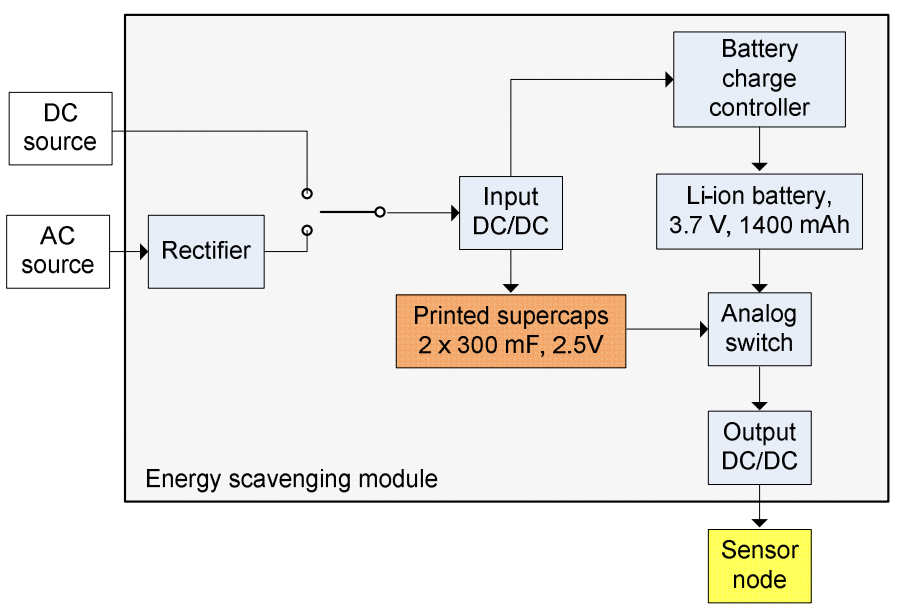

Fig. 2. The energy scavenging module architecture 
In this first prototype, to initiate the ESM operation a user must specify by a "jumper" which type (DC or AC) of environmental energy is available for harvesting. DC type is routed directly to the input DC/DC which supports up to $11 \mathrm{~V}$ of input voltage. Conversely, when the AC based ambient source is selected, the ESM rectifies the signal and provides the input DC/DC with it. The voltage rectifier is composed of a diode in conjunction with a polar capacitor. The input DC/DC operates as a $5 \mathrm{~V}$ generator to supply the system. The system, in turn, has several options for input using the input voltage: it can charge the printed capacitors, charge the Li-ion battery or supply the load. If the printed capacitors are charged they function as a filter. The battery charge controller performs the proper and accurate charging of $3.7 \mathrm{~V}, 1400 \mathrm{mAh} \mathrm{Li}$ ion battery. The analog switch allows the system to choose either ambient power source with/or the printed caps or Li-ion battery as a power supply for a sensor node. To provide a sensor node platform with a stable voltage, the output DC/DC generates $3 \mathrm{~V}$. The ESM requires an external control signal for the analog switch and provides two analog-to-digital (ADC) pins for a sensor node to monitor the voltage level of the printed caps and the Li-ion battery.

\subsection{ESM Design}

The energy scavenging board (see Figure 3) was designed as a 4-layer PCB. Since the system supports (AC) and (DC) based ambient sources, the AC-to-DC voltage conversion is performed by a standard diode rectifier and an electrolytic capacitor. Further stabilization of the rectified DC voltage or ambient DC voltage to $4.6 \mathrm{~V}$ is achieved by a MAX1672 step-up/down DC/DC converter.

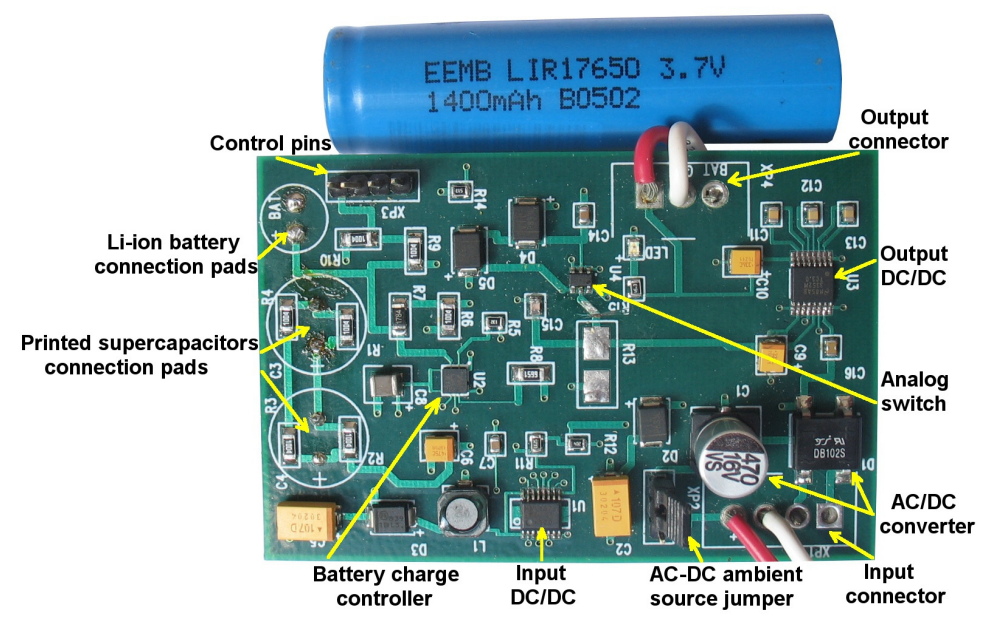

Fig. 3. Photograph of the energy scavenging module

The primary energy buffer as the printed super capacitors is connected in parallel to the $4.6 \mathrm{~V}$ terminal. The printed capacitor implementation is described in Section 4.3 in details. The series wiring of two super capacitors requires balancing to guarantee equal 
voltage sharing. We applied passive balancing to maintain similar voltages between the super capacitors, where the leakage currents may be slightly different. Passive balancing uses equal value resistors (of the order of several MOhms) in parallel with the super capacitors to let a small current flow between the super capacitors, thus keeping the voltages at the same level.

The secondary energy buffer functions are performed by a EEMB LIR17650 Liion rechargeable battery having 3.7 nominal voltage and 1400mAh typical capacity. The Li-ion battery requires a complex charging circuit, e.g., a special charge controller. In our case we used the integrated MIC79110 battery charge controller. The secondary buffer is charged by the primary buffer. However, with the primary buffer being charged and the ambient power source providing stable voltage, the secondary buffer can be charged directly. A Single Pole Double Throw (SPDT) analog switch MAX4624 having high current carrying capability and low power consumption is used to choose between (switching speed is $50 \mathrm{~ns}$ maximum) the primary and secondary power buffer to supply the wireless sensor node. The energy scavenging module output voltage of $3.0 \mathrm{~V}$ is provided using the LM3352 step-up/down DC/DC converter which provides voltage stabilization. In order to ensure interconnection between the ESM and the wireless sensor node, we have used a 4-pin connector for charge/monitor battery control, super capacitor voltage monitoring and analog switch control.

\subsection{Printed Capacitors Implementation}

Electrode slurries are composed of 50 wt. $\%$ activated carbon with 24 wt. $\%$ PVDF polymer binder, 2 wt. $\%$ conductive carbon black additives, and $24 \mathrm{wt} . \% \mathrm{BMIM}^{+} \mathrm{BF}_{4}{ }^{-}$ ionic liquid. Electrolyte gels are a 1:1 composite of PVDF and $\mathrm{BMIM}^{+} \mathrm{BF}_{4}^{-}$. Current collector slurries of conductive particles (such as nickel or carbon) in PVDF are also being developed. The viscosities of the slurries are tailored by adding a volatile solvent such as n-methyl-2-pyrollidone (NMP). The pneumatic dispenser printer extrudes a rapid succession of drops through a syringe tip onto the substrate. The drops eventually coalesce to form a film as NMP is removed via drying. To fabricate a capacitor, a five-layer planar and symmetric sandwich of current collector, carbon electrode, and electrolyte ink are printed as shown in Figure 4. Figure 5 displays a micrograph showing the material morphology of the electrode and electrolyte.

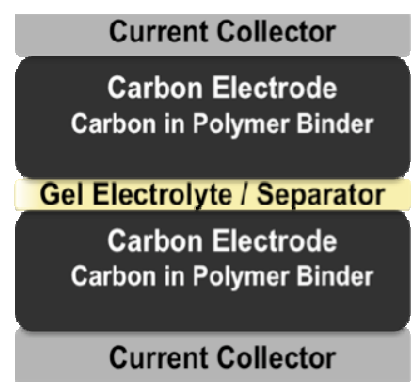

Fig. 4. Schematic of a carbon electrochemical capacitor 


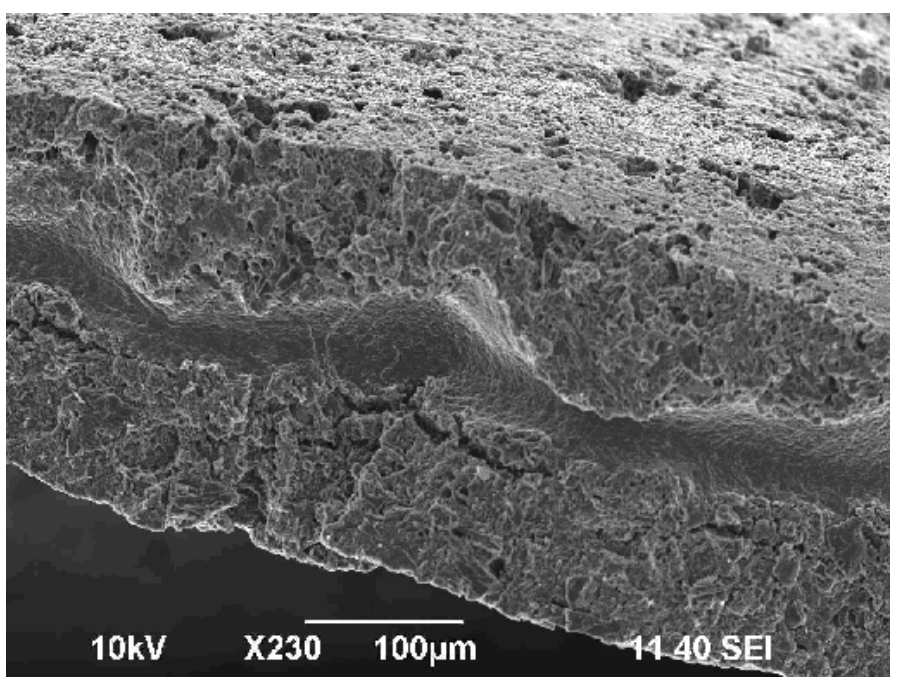

Fig. 5. Micrograph showing the morphology of the carbon electrode and electrolyte active layers

To deposit supercapacitors on a substrate already populated with other components, automated alignment software and image processing are used to determine any open spaces available for printing. An image is taken of the substrate and any open spaces are translated into a bitmap image. The printer then deposits material in the pattern of the bitmap, and the alignment software maintains accurate orientation in between the printing of films even with the adjustment of syringe tips, substrate rotation, or other printing parameters. In this study, capacitor modules were tested rather than integrated cells, and each module was clamped to maintain the interfaces adjacent to each printed layer.

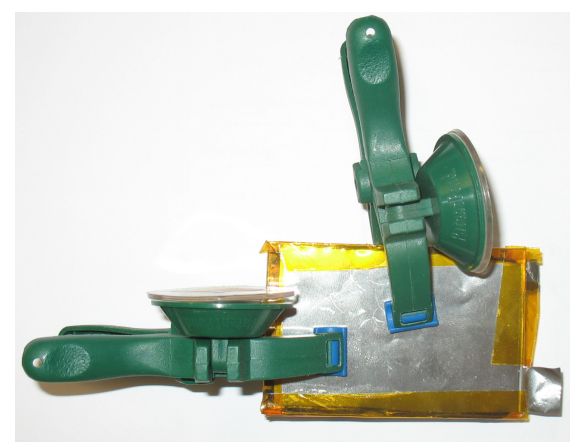

a)

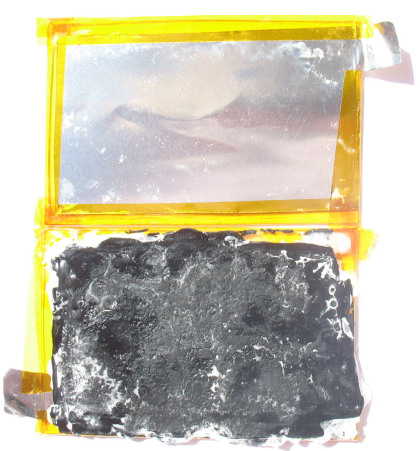

b)

Fig. 6. The photographs of printed caps (a) separate printed supercap sample $5 \times 7.5 \mathrm{~cm}$, (b) a look inside the supercap 
Figure 6 demonstrates the stand alone printed supercapacitor and its internal chemistry. Table 1 demonstrates the technical specification of the supercap used in this work.

Table 1. Technical specification of the supercap used in this work

\begin{tabular}{l|l} 
Parameter & Value \\
\hline Capacity, $\mathrm{mF} / \mathrm{cm}^{2}$ & $10-40$ \\
Power density, $\mu \mathrm{W} / \mathrm{cm}^{2}$ & 575 \\
Maximum voltage, $\mathrm{V}$ & 2.5 \\
$\begin{array}{l}\text { Maximum charge/discharge current, } \mathrm{mA} \\
\text { Life cycles }\end{array}$ & 2 \\
$\begin{array}{l}\text { Charging time with office light, } \\
\text { minutes }\end{array}$ & $>120,000$ \\
$\begin{array}{l}\text { Charging time with solar radiation, } \\
\text { minutes }\end{array}$ & $\sim 6$ \\
Footprint, cm & $5 \times 7.5$ \\
\hline
\end{tabular}

Figure 7 shows the supercapacitor behaviour (energy storage per $\mathrm{cm}^{2}$ and selfdiscarge capability) in ten charge/discharge cycle. We would like to note that after approximately 100 charge/discarge cycles the capacity of the cell can improve. Besides, better capacity and low leakage current can be achieved with proper stressing of the cell's plates. The application of passive balancing explained in Section 4.2 may help to decrease leakage current as well.

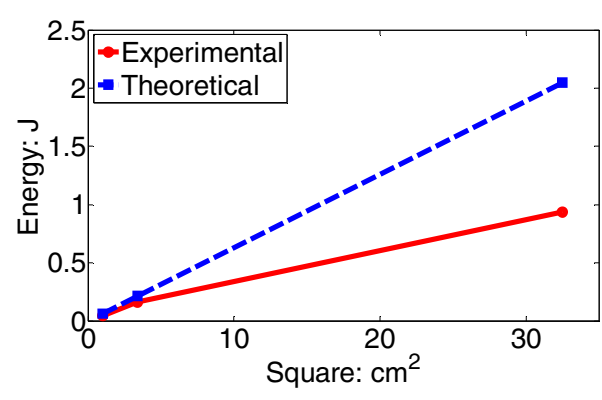

a)

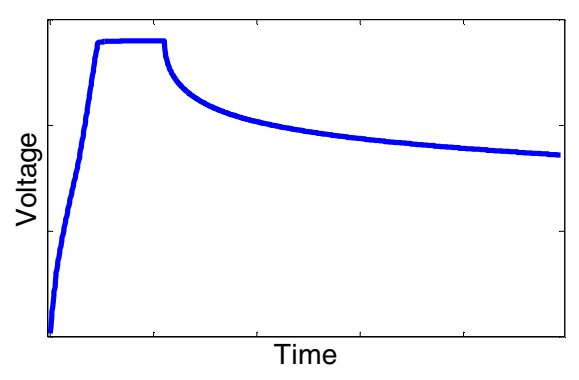

b)

Fig. 7. Supercapacitor behaviour after 10 charge-discharge cycles (a) energy storage per $\mathrm{cm}^{2}$, (b) example of charge-discharge curve involving self-discharge

The experimental curve shown in Figure 7a was obtained by measuring the size, capacity and voltage of three samples, and by further computing the stored energy using $E=\left(C U^{2}\right) / 2$, where $E$ is the energy stored in the capacitor, and $C$ and $U$ are its capacity and voltage, respectively. The theoretical curve shows that the energy 
capacity of the capacitor is directly proportional to its footprint. Figure $7 \mathrm{~b}$ shows an example curve of the supercapacitor self-discharge without passive balancing.

\section{$5 \quad$ Experimental Results}

The main purpose of this section is to evaluate the printed supercapacitors potential to operate as the energy buffer for the sensor node. The developed ESM supplies a sensor node using AC- and DC-based harvesters. For our experiments we have chosen a TelosB sensor node which was programmed to measure temperature, humidity and send the measured data to a gateway once each $5 \mathrm{~s}$. In order to evaluate the battery performance in short time we have used a $20 \%$ duty cycle. In real deployments, however, the duty cycle must be as low as possible, e.g. 1-10\%, to minimize the depletion of energy buffers. This experiment is carried out during 24 hours: 12 hours with the AC harvester and 12 hours with the DC one. This experiment is focused on the superacapacitors and does not cover maximum power point tracking [22], [24] for the harvesters.

We start our experiments with the harvesting of AC-based signal. We do not have real AC harvester available and, therefore, simulate one using a signal generator and current divider. Figure 8 shows the experimentation test-bed in our electronics lab. The following simulation parameters for the AC harvester are adopted from [21]: voltage $(V)=5 \mathrm{~V}$, frequency $(f)=120 \mathrm{~Hz}$, generated power $\left(P_{\text {out }}\right)=277 \mathrm{uW}$ (per 0.5 $\mathrm{cm}^{3}$ of harvesting material). Due to the physical limitations of the printed supercapacitor technology (discharge current no more than $2 \mathrm{~mA}$ ) they were only used for sensing and sleep mode. Since communication on the TelosB mote requires $\sim 23 \mathrm{~mA}$ in active mode it was supported by the Li-ion battery.

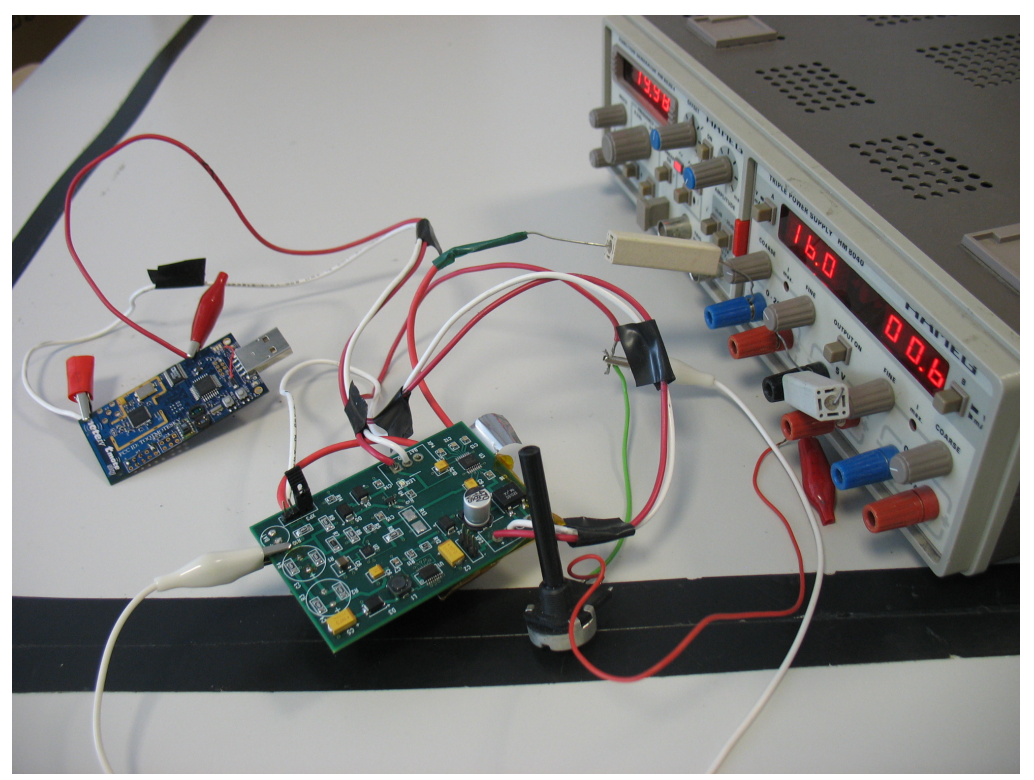

Fig. 8. Test bed for printed supercapacitors evaluation using simulated AC-based harvester 
Figure 9 shows that the experiment with the simulation of the AC harvester lasts 12 hours (let say from $8 \mathrm{pm}$ for our convenience). The AC rectifier circuit provides a stable $5.7 \mathrm{~V}, 55 \mathrm{uA}$ charging signal. The supercapacitors are discharged to $1.36 \mathrm{~V}$ in approximately 20 minutes. This voltage level can not supply the ESM circuit. The system fully switches to the Li-ion battery as the main power supply. The battery had $4.03 \mathrm{~V}$ before the experimentation. During the whole 12 hours it was discharged to $3.8 \mathrm{~V}$ which equals to a loss of $10 \%$ of its nominal capacity. The low discharge threshold for this cell is $2.7 \mathrm{~V}$. After the 12-hour period, we assume that the DC-based harvester can provide more ambient energy, e.g. solar radiation, and, therefore, switch the ESM to DC harvesting mode. It should be noted, however, that the sensor node was operating with $20 \%$ duty cycle.

At $1 \%$ duty cycle (see Figure 9 ) the sensor node can perform the sensing almost for 8 hours which is promising for the future WSNs deployment. Besides, during this experiment we stressed the plates of the supercapacitors much better (see Section 4) than in case of $20 \%$ duty cycling. This resulted in approximately 8 hours of operation instead of expected approximately 6.5 hours. $1 \%$ duty cycle experiment, therefore, shows that current ink printing fabrication technology allows the most efficient supercapacitors operation (in terms of lifetime) at low duty cycles.
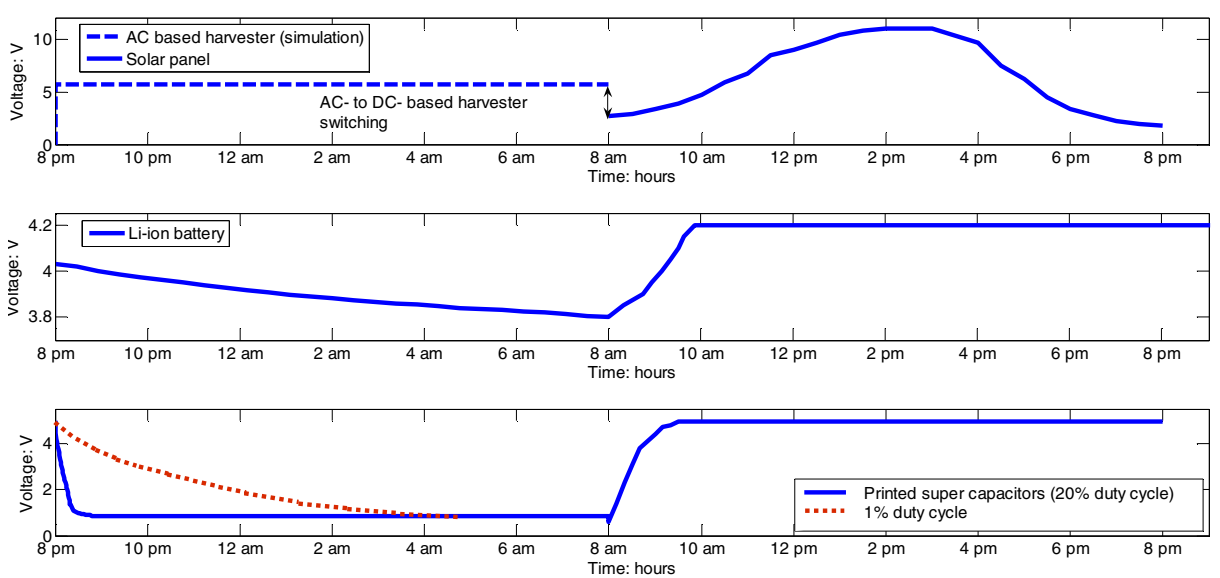

Fig. 9. Status of the harvesters, Li-ion battery, and printed supercapacitors during 24-hours deployment

Starting from 8 am in the morning we deploy our system in the garden (see Figure 10). We put the TelosB mote and the ESM near the shrub and locate the harvester, a BP SX305M solar panel, a bit higher so that it could scavenge direct light. The charging status of the supercapacitors and the Li-ion battery strongly depends on weather conditions. Since the experiment is carried out in August the panel generates sufficient power for charging purposes and direct supply of the TelosB mote.

The aim of this deployment is the experimentation in real conditions. We check if there is enough light for system operation and how the system behaves even when 
shadow, for instance, covers the solar panel, but we do not change the position of harvester during the time of experiment. Figure 9 shows that the harvester generates a sufficient amount of current for the TelosB operation and for charging both energy buffers. However, solar radiation in the morning does not provide high current for fast buffers replenishment. That is why it takes around 90 minutes to fully charge the supercapacitors and around 2 hours to charge the battery to its maximum $4.2 \mathrm{~V}$ level and full capacity. The experiment was carried out in typical weather conditions. It should be noted that during overclouded days the battery will be fully charged as well, but it will obviously take longer.

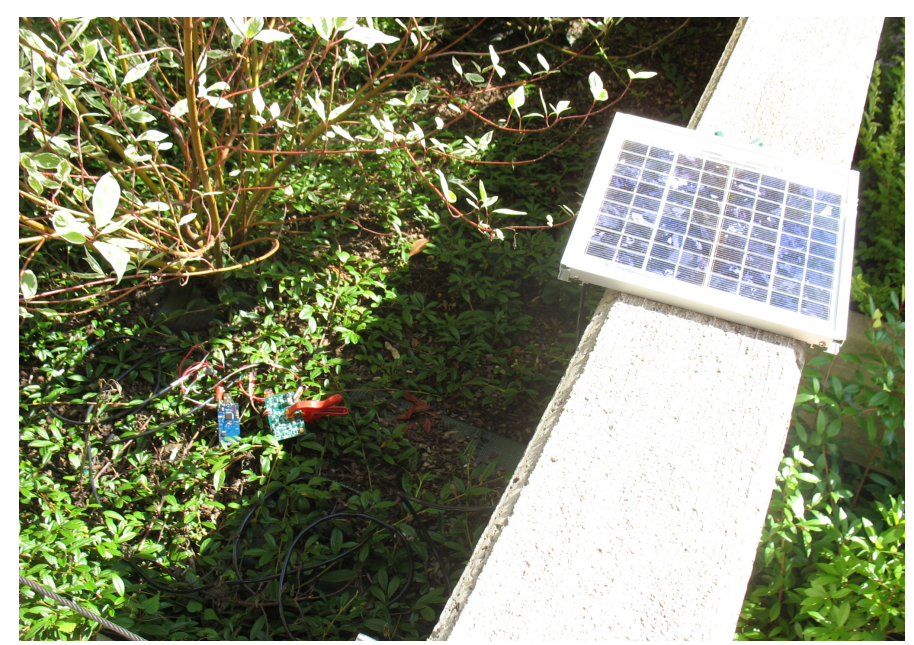

Fig. 10. The system deployment with DC-based harvester

The experiment shows that at this workload and with the AC harvester only the system will go down in five days. In order to prolong the lifetime of the mote a user must decrease the duty cycle (at least turn on the radio rarely because it consumes high power) or use more powerful harvester. The application of DC harvester demonstrates potentially 'perpetual' operation of the mote. The user, however, should carefully calculate the availability of solar radiation in the region of network deployment, calculate maximum power point tracking, and consider overcast days as well.

\section{$6 \quad$ Related Work}

In this section we review the recent development of wireless sensor node platforms utilizing energy scavenging technology and discuss how their energy buffer(s) arranged.

Because of the performance and lifetime demands of WSNs, energy scavenging technology [10] has been researched heavily to take advantage of available on-site energy. By implementing energy scavenging, the sensor node lifetime can be effectively increased. Table 2 summarizes popular sensor node platforms with energy scavenging technology. 
PicoCube [6], VIBES [8] and PMG Perpetuum [7] are wireless sensor platforms powered via ambient vibrations. PicoCube is a low power, $1 \mathrm{~cm}^{3}$ sensor node designed for tire pressure monitoring. The node contains five printed circuit boards packed in a plastic case. Being fully autonomous, PicoCube does not require an external control. A NiMH battery and an electromagnetic shaker energy harvester enable long-term operational support to the platform.

Table 2. Popular WSN platforms with energy scavenging technology

\begin{tabular}{l|l|l|l} 
Platform & $\begin{array}{l}\text { Ambient } \\
\text { source }\end{array}$ & $\begin{array}{l}\text { Energy buf- } \\
\text { fer }\end{array}$ & Purpose \\
\hline Everlast [2] & Solar radiation & Primary & $\begin{array}{l}\text { Operation on super capaci- } \\
\text { tors only }\end{array}$ \\
\hline Prometheus [3] & Solar radiation & Dual & $\begin{array}{l}\text { Proof of concept: 'perpetual } \\
\text { operation' }\end{array}$ \\
\hline ZebraNet [4] & Solar radiation & Secondary & Zebras' movement tracking \\
\hline Heliomote [5] & Solar radiation & Secondary & Ecosystem sensing \\
\hline PicoCube [6] & Vibration & Secondary & Tire pressure monitoring \\
\hline $\begin{array}{l}\text { PMG Perpe- } \\
\text { tuum [7] }\end{array}$ & Vibration & Primary & Battery-free sensor system \\
\hline VIBES [8] & Vibration & Primary & Acceleration data measuring \\
\hline $\begin{array}{l}\text { AmbiMax [9] } \\
\text { Vibration, solar }\end{array}$ & Dual & $\begin{array}{l}\text { Multiple harvesting compo- } \\
\text { nents adoption }\end{array}$ \\
\hline $\begin{array}{l}\text { Electro acous- } \\
\text { tic liner system } \\
\text { [20] }\end{array}$ & Acoustic noise & Secondary & $\begin{array}{l}\text { Suppression of aircraft en- } \\
\text { gine noise }\end{array}$ \\
\hline
\end{tabular}

The intended application for the VIBES sensor node is an air compressor unit, though the vibrations and frequencies measured pointed out opportunities for different industrial applications. The microsystem contains three main units: a microgenerator which converts ambient vibration energy into electrical energy, a voltage multiplier which converts and stores the energy in a supercapacitor, and a microcontroller based subsystem consisting of an accelerometer and an RF transmitter. VIBES is an energy aware system that can potentially adjust its duty cycle according to the available energy.

The third platform, PMG from Perpetuum, is based on a vibration energy harvesting microgenerator. The PMG node has three types of sensor nodes, each developed for a particular application; PMG-17 [7] is intended for control of plant machinery and continuous monitoring, PMG-27 [7] is oriented to the aerospace industry, and PMG-37 [7] was developed for transportation applications. PMG platforms have a primary energy buffer for improved flexibility and do not require any maintenance. The PMG-17 energy harvester, for instance, can generate around $50 \mathrm{~mW}$ at $1 \mathrm{~g}$ and can operate continuously, and is especially attractive for very data intensive applications. Depending on the appropriate application, the PGM node can be configured to transmit large data bursts over low duty cycles, or transmit smaller data burst more frequently, for example, for data in a dynamic environment. However, PMG platform 
made of metal is, obviously, much heavier than typical devices based on printed circuit board technology. Besides, the primary buffer (supercapacitors) has significant discharge current [3]. In case of sudden unavailability of a vibration source the energy buffer can be discharged quickly.

To avoid using rechargeable batteries which are limited in lifetime, Everlast [2], like the two previous platforms, utilizes a primary energy buffer of supercapacitors. These supercapacitors can be efficiently charged using a pulse frequency modulated (PFM) converter and an open-circuit solar voltage approach to track the maximum power point. The operation lifetime of Everlast is predicted as 20 years, requiring no maintenance. This platform as well as PMG one due to high leakage current of supercapacitors is highly dependable on energy source availability.

Prometheus [3] is a wireless sensor platform which includes the Prometheus power board and the Telos Micro Controller Unit (MCU). The entire system is powered by the Prometheus power board. This power board is implemented with a two-stage storage system containing two supercapacitors as a primary buffer and a lithium rechargeable battery as a secondary buffer. The two supercapacitors are wired in series to decrease leakage current. The lithium battery is charged only from the primary buffer when energy is stable and pulsing is possible. The control software of the Prometheus platform is pushed to the Telos MCU to reduce the number of components on the board and thus decrease power consumption and board space. The developers of this system claim 43 years operation under a $1 \%$ duty cycle, 4 years under $10 \%$ loading, and 1 year under $100 \%$ loading [3].

ZebraNet [4] is a Global Positioning System (GPS) based WSN for animal migration tracking with sensors built into the collars of the necks of zebras. The wireless sensor node has the following hardware features: lithium-ion battery single energy buffer, dualclock microcontroller, separate power supplies for each device to lower energy consumption, and an off-chip memory since the system has to generate detailed logs. The middleware of the system has GPS sensing and radio communication as priority events, and the remaining events could be regarded as a combination of scheduled and unplanned events. To reduce power consumption developers carried out three low-level techniques: timely use of components, on-the-fly processing, and dual clock scheme which consumes half the energy compared to the use of a slow clock.

The Heliomote [5] platform contains an off-the-shelf Mica2 module [23]. Heliomote was assumed to be a plug-and-play enhancement to the motes for environmental monitoring. The platform solely utilizes a NiMH rechargeable battery as its energy buffer. Hardware support for the battery long term operation have power routing switches that provide overcharge and undercharge protection, and an on-board power monitoring integrated circuit. Because of the inherent leakage and increased overhead of energy storage management, developers renounced exploiting the super capacitors.

Self-powered electroacoustic liner system [20] was designed specifically for the suppression of aircraft engine noise. The module consists of three subsystems: energy harvesting, communication, and impedance tuning. One of two electromechanical Helmholtz resonators (EMHR) is assigned for energy harvesting whereas the second one for noise suppression. However, more EMHRs can be used if more power is required to supply the system. The designed module has a secondary energy buffer as a rechargeable battery. 
Finally, AmbiMax [9] enables to harvest ambient wind, vibrations, solar radiation and thermal energy. The platform has a primary energy buffer. However, the AmbiMax designers make an emphasis on maximum power point tracking that results in efficient system operation as well as in the efficient charging of the supercapacitors.

Of the nine platforms with energy scavenging technology that we have considered, solely the AmbiMax is able to support multiple harvesters which are predefined for the platform. However, the realization of ubiquitous WSN concept based on AmbiMax platform seems as highly expensive solution due to adopting many harvesters of different kind. The remaining systems that we have surveyed have an integrated, fixed harvesting component. Most of the sensor platforms have a primary energy buffer as a supercapacitor(s) in standard package and some of them have secondary energy buffer as a rechargeable battery.

\section{Conclusions and Future Work}

In this work we proposed the application of printed supercapacitors (fabricated using 'direct write' technology) as an energy buffer of a sensor node to improve its longterm operation. The 'direct write' technology can potentially provide the efficient use of free space of the board and manufacture the capacitors with required parameters.

For our experiments we designed an Energy Scavenging Module (ESM) which supplies the sensor node with ambient AC- or DC-based energy. The surplus of scavenged ambient energy is stored in two energy buffers. As the primary buffer we used the printed capacitors. The experiments ensure the printed capacitors operate well at low duty cycles, e.g. at $1 \%$ duty cycle the TelosB can perform sensing for almost 8 hours, but due to technological limitations can support charge/discharge current which does not exceed $2 \mathrm{~mA}$. It should be noted that for achieving the best capacity and low leakage currents, the plates of the printed storage must be stressed properly.

During the printing of the supercaps on the board material we discovered some issues with cracking on a board surface: it is very slick and many of polymers are peeling off. That is why the first ESM version supports the printed supercaps that are physically clipped to the board. To address this problem in the future we may have to chemically etch the surface of the board in order to create some sort of roughness, then the polymer may stick better.

\section{References}

[1] Selavo, L., Wood, A., Cao, Q., SooKoor, T., Liu, H., Srinivasan, A., Wu, Y., Kang, W., Stankovic, J., Young, D., Porter, J.: LUSTER: Wireless Sensor Network for Environmental Research. In: 5th International Conference on Embedded Networked Sensor Systems, Sydney, Australia, pp. 103-116 (2007)

[2] Simjee, F., Chou, P.H.: Everlast: Long-life, Supercapacitor-operated Wireles Sensor Node. In: International Symposium on Low Power Electronics and Design, Tegernsee, Germany, pp. 197-202 (2006)

[3] Jiang, X., Polastre, J., Culler, D.: Perpetual Environmentally Powered Sensor Networks. In: 4th International Symposium on Information Processing in Sensor Network, pp. 463468 (2005) 
[4] Zhang, P., Sadler, C.M., Lyon, S.A., Martonosi, M.: Hardware Design Experience in ZebraNet. In: 2nd International Conference on Embedded Networked Sensor Systems, Baltimore, USA, pp. 227-238 (2004)

[5] Raghunathan, V., Kansal, A., Hsu, J., Friedman, J., Srivastava, M.: Design Considerations for Solar Energy Harvesting Wireless Embedded Systems. In: 4th International Symposium on Information Processing in Sensor Networks, pp. 457-462 (2005)

[6] Chee, Y.H., Koplow, M., Mark, M., Pletcher, N., Seeman, M., Burghardt, F., Steingart, D., Rabaey, J., Wright, P., Sanders, S.: PicoCube: A $1 \mathrm{~cm}^{3}$ Sensor Node Powered by Harvested Energy. In: Design Automation Conference, Anaheim, USA, pp. 114-119 (2008)

[7] Perpetuum, http: / / www . perpetuum.co.uk

[8] Torah, R.N., Tudor, M.J., Patel, K., Garcia, I.N., Beeby, S.P.: Autonomous Low Power Microsystem Powered by Vibration Energy Harvesting. In: 6th Annual IEEE Conference on Sensors, Atlanta, USA, pp. 264-267 (2007)

[9] Park, C., Chou, P.H.: AmbiMax: Autonomous Energy Harvesting Platform for MultiSupply Wireless Sensor Nodes. In: 3rd Annual IEEE Communications Society on Sensor and Ad Hoc Communications and Networks, vol. 1, pp. 168-177 (2006)

[10] Roundy, S., Wright, P.K., Rabaey, J.M.: Energy Scavenging for Wireless Sensor Networks: With Special Focus on Vibrations. Kluwer Academic Publishers Group, Boston (2003)

[11] Simon, P., Gogotsi, Y.: Materials for Electrochemical Capacitors. Nature Materials 7 , 845-854 (2008)

[12] Kotz, R., Carlen, M.: Principles and Applications of Electrochemical Capacitors. Electrochimica Acta 45, 2483-2498 (2000)

[13] Conway, B.: Electrochemical Supercapacitors, Scientific Fundamentals and Technological Applications. Kluwer Academic, New York (1999)

[14] Lewandowski, A., Galiński, M.: Carbon - Ionic Liquid Double-Layer Capacitors. Physics and Chemistry of Solids 65, 281-286 (2004)

[15] Forsyth, S.A., Pringle, J.M., MacFarlane, D.R.: Ionic Liquids - an Overview. Australian Journal of Chemistry 57, 113-119 (2004)

[16] Ho, C.C., Steingart, D.A., Evans, J.W., Wright, P.K.: Tailoring Electrochemical Capacitor Energy Storage Using Direct Write Dispenser Printing. ECS Transactions 16(1), 35-47 (2008)

[17] Hart, J.P., Wring, S.A.: Recent Developments in the Design and Application of ScreenPrinted Electrochemical Sensors for Biomedical, Environmental and 115 Industrial Analyses. TrAC Trends in Analytical Chemistry 16(2), 89-103 (1997)

[18] Arnold, C.B., Pique, A.: Laser Direct-Write Processing. Mrs Bulletin 32, 9-12 (2007)

[19] Steingart, D.A., Ho, C.C., Salminen, J., Evans, J.W., Wright, P.K.: Dispenser Printing of Solid Polymer - Ionic Liquid Electrolytes for Lithium Ion Cells. In: 6th International IEEE Conference on Polymers and Adhesives in Microelectronics and Photonics, Oadaiba, Tokyo, Japan, pp. 261-264 (2007)

[20] Phipps, A., Liu, F., Cattafesta, L., Sheplak, M., Nishida, T.: Demonstartion of a Wireless, SelfPowered, Electroacoustic Liner System. Acoustic Society of America 125(2), 873-881 (2009)

[21] Roundy, S., Wright, P.K., Rabaey, J.: A Study of Low Level Vibrations as a Power Source for Wireless Sensor Node. Computer Communications (Elsevier) 26, 1131-1144 (2003)

[22] Raghunathan, V., Chou, P.H.: Design and Power Management of Energy Harvesting Embedded Systems. In: Internationsl Symposium on Low Power Electronics and Design, Togernsee, Germany, pp. 369-374 (2006)

[23] Memsic, Mica2 mote, http: / /www. memsic. com

[24] Brunelli, D., Moser, C., Thiele, L., Benini, L.: Design of a Solar-harvesting Circuit for Batteryless embedded Systems. IEEE Transactions on Circuits and Systems I: Regular papers 56(11), 2519-2528 (2009) 